improve their ability to spot targets on the unattended side while they focus their attention on the side indicated by the arrow. This recovery seems to depend mostly on the right temporal parietal junction, a brain area thought to be responsible for interrupting attention and allowing a shift of attention toward the target location. This area is active only in the right hemisphere during shifts of attention to targets in either direction, whereas most other areas are symmetric ${ }^{6}$. The right lateralization of this area seems to be a major reason for the strong neglect of the left side apparent in the participants' performance (Fig. 1).

The Corbetta et al. study ${ }^{1}$ reveals that damage to the ventral areas of the spatial attention system produces neglect because it causes dysfunction of the dorsal system as well. Although this was not a specific focus of the current study, the results raise the question of why more dorsal lesions do not cause malfunctions of the ventral parietal area and thus also produce neglect. Apparently, there is an asymmetry between the ventral and dorsal parietal areas: lesions of the dorsal parietal area alone do not cause neglect ${ }^{7}$. The mechanisms underlying this strong asymmetry in the remote effects of these two critical areas of the parietal lobe remain unclear.

Evidence for greater than normal left hemisphere activity during the acute stage of neglect provides some basis for rehabilitation methods as well. The authors suggest that competition between hemispheres may provide the basis for helping recovery, either by increasing activity in the ipsilesional cortex or by reducing it in the contralesional cortex. Increased right hemisphere activity through warning signals, already known to reduce neglect, could serve as one method for improving hemispheric balance ${ }^{8}$. The need for hemispheric balance could be an important reason why forcing the increased use of a paralyzed limb can foster recovery ${ }^{9}$. Among patients with neglect, those with right hemisphere lesions, who show a chronic rightward bias, may also be helped by inhibiting left superior parietal lobe activity ${ }^{1}$.

There are more general lessons that might be gleaned from this work. Remote effects of lesions provide some explanation of how local- ized computations, as revealed in many functional imaging studies, may be consistent with the occurrence of a syndrome with many complex sensory and motor features. This finding may be important for all of neuropsychology. The authors also argue that the general principles of recovery that they have found might apply to aphasia or sensory motor deficits. In any case, the paper provides a strong argument for using imaging during recovery to determine the effects of various forms of therapy.

1. Corbetta, M., Kincade, M.J., Lewis, C. \& Sapir, A. Nat Neurosci. 8, 1603-1610 (2005).

2. Corbetta, M. \& Shulman, G.L. Nat. Rev. Neurosci. 3 201-215 (2002).

3. Posner, M.I. Q. J. Exp. Psychol. 32, 3-25 (1980)

4. Posner, M.I., Walker, J.A., Friedrich, F.J. \& Rafal, R.D. J. Neurosci. 4, 1863-1874 (1984).

5. Hillyard, S.A., DiRusso, F. \& Martinez, A. in Functional Neuroimaging of Visual Cognition (eds. Kanwisher, N. \& Duncan, J.) 381-388 (Oxford Univ. Press, Oxford, 2004).

6. Perry, R.J. \& Zeki, S. Brain 123, 2273-2288 (2000).

7. Friedrich, F.J., Egly, R., Rafal, R.D. \& Beck, D. Neuropsychology 3, 193-207 (1998).

8. Robertson, I.H., Mattingley, J.B., Rorden, C. \& Driver, J. Nature 395, 169-172 (1998).

9. Taub, E., Uswatte, G. \& Elbert, T. Nat. Rev. Neurosci. 3, 228-236 (2002).

\title{
Time to smell the roses
}

Timing is a thorny issue for the chemical senses. Principal neurons in the vertebrate olfactory bulb and insect antennal lobe have dynamic odor-evoked responses that can long outlast odorant exposure. The temporal pattern of these responses is thought to be important for distinguishing different odorants, but in a natural environment, odor stimuli have their own temporal structure. With an animal's movements and the intermittent arrival of odors in the air, new odors are likely to interrupt responses to previous ones. Experience tells us that a second sniff of a rose still smells like a rose, but how does the olfactory system sort out these potentially conflicting time courses?

On page 1568 of this issue, Mark Stopfer and colleagues address this question in the olfactory system of the locust. They exposed adult locusts to trains of brief odorant pulses with natural interpulse intervals, and made intracellular and extracellular recordings from projection neurons (PNs) in the antennal lobe. In most cases, responses varied over successive pulses, showing that the temporal structure of odorant presentation did influence the temporal pattern of PN responses.

To determine the downstream consequences of this interference, the authors considered known features of the locust olfactory system. Each antennal lobe contains 830 PNs, and more than 100 PNs converge onto each of about 50,000 Kenyon cells in the mushroom body, a brain area important for olfactory memory. Odorant stimulation evokes oscillations in the antennal lobe, and Kenyon cells seem to integrate convergent PN input over 50 ms, approximately one oscillation cycle. The authors pooled 117 PN recordings from multiple experiments and constructed activity vectors over 50-ms windows as well as ensemble activity trajectories over the entire PN responses to different patterns of odor pulses.

In contrast to the varied responses in individual PNs, ensemble PN activity showed highly similar responses to multiple pulses. Response trajectories for repeated odor pulses overlapped, with successive pulses appearing to partially reset the circuitry such that each response began similarly and followed a similar trajectory, irrespective of the pulse timing. A template chosen from any $50-m s$ window of a response could accurately classify odorant identity for responses in different presentation patterns.

These results suggest that the problem of temporal interference may be adequately solved by converging PN input onto Kenyon cells, which also responded reliably to repeated odorant pulses. However, this solution depends on the ability of newly arriving odors to reset ensemble activity in the antennal lobe. What restricts PNs to repeatedly return to the same response trajectory despite ongoing dynamic activity? Can an ongoing response to one odorant also be reset by the arrival of a different smell? Researchers undoubtedly will continue to sniff around for these answers.

Cara Allen 